\title{
Detailed Oil Compositional Analysis Enables Evaluation of Impact of Temperature and Biomass-to-Catalyst Ratio on ex Situ Catalytic Fast Pyrolysis of Pine Vapors over ZSM-5
}

Himanshu Patel ${ }^{\dagger 1}$, Naijia Hao ${ }^{\dagger^{2}}$, Kristiina Iisa ${ }^{3 *}$, Richard J. French ${ }^{3}$, Kellene A. Orton ${ }^{3}$, Calvin Mukarakate ${ }^{3}$, Arthur J. Ragauskas $^{2,4,5^{*}}$, Mark R. Nimlos ${ }^{3}$

${ }^{1}$ Process Design \& Engineering Cell, CSIR-Central Salt \& Marine Chemicals Research Institute, Shree Gijubhai Badheka Marg, Bhavnagar 364002, Gujarat, India

${ }^{2}$ Department of Chemical and Biomolecular Engineering, University of Tennessee, Knoxville, 1512 Middle Dr, Tennessee 37996, United States

${ }^{3}$ National Renewable Energy Laboratory, 15013 Denver West Parkway, Golden, CO 80401, United States

${ }^{4}$ Joint Institute of Biological Science, Biosciences Division, Oak Ridge National Laboratory, 1 Bethel Valley Rd, Oak Ridge, Tennessee 37831, United States

${ }^{5}$ Center for Renewable Carbon, Department of Forestry, Wildlife, and Fisheries, University of Tennessee Institute of Agriculture, Knoxville, Tennessee 37996, United States

${ }^{\dagger}$ Equal contribution from both authors.

*Corresponding author: aragausk@utk.edu

kristiina.iisa@nrel.gov 


\section{Number of Pages: 15}

\section{Figures}

Figure S1. XRD analysis of the fresh ZSM-5 catalyst and post-reaction catalysts from the CFP experiments.

Figure S2. 2-inch fluidized bed reactor system with ex situ upgrading with continuous catalyst feed and removal.

Figure S3. TGA traces for fresh catalyst and coked catalysts. The fresh catalyst shows the presence of $8 \%$ of water or other volatile material. For the coked catalysts, the mass loss up to $\sim 250^{\circ} \mathrm{C}$ was attributed to water and other loosely adsorbed species, and the mass loss after $650^{\circ} \mathrm{C}$ to coke.

Figure S4. Yield of oxygen in the CFP products as a function of a) biomass:catalyst mass ratio and b) upgrading temperature.

Figure S5. Effect of upgrading temperature on aliphatic $\mathrm{C}-\mathrm{H}$ bonds in the HSQC NMR spectra for (a) $500^{\circ} \mathrm{C} \mathrm{B:C}$ 1.4 bottom, (b) $550^{\circ} \mathrm{C} \mathrm{B:C} 1.4$ bottom, (c) $600^{\circ} \mathrm{C} \mathrm{B:C} 1.4$ bottom, (d) $500^{\circ} \mathrm{C} \mathrm{B:C} 1.4$ top, (e) $550^{\circ} \mathrm{C} \mathrm{B:C} 1.4$ top, and (f) $600^{\circ} \mathrm{C}$ B:C 1.4 top.

Figure S6. Effect of B:C ratio on aliphatic $\mathrm{C}-\mathrm{H}$ bonds in the HSQC NMR spectra for (a) $550^{\circ} \mathrm{C} \mathrm{B}: \mathrm{C} 1.0$ bottom, (b) $550^{\circ} \mathrm{C} \mathrm{B:C} 1.4$ bottom, (c) $550^{\circ} \mathrm{C} \mathrm{B:C} 1.8$ bottom, (d) $550^{\circ} \mathrm{C} \mathrm{B:C} 1.0$ top, (e) $550^{\circ} \mathrm{C} \mathrm{B:C} 1.4$ top, and (f) $550^{\circ} \mathrm{C}$ B:C 1.8 top.

Figure S7. Effect of upgrading temperature on aromatic C-H bonds in the HSQC NMR spectra for (a) 500 ${ }^{\circ} \mathrm{C}$ B:C 1.4 bottom, (b) $550^{\circ} \mathrm{C} \mathrm{B:C} 1.4$ bottom, (c) $600^{\circ} \mathrm{C} \mathrm{B:C} 1.4$ bottom, (d) $500^{\circ} \mathrm{C} \mathrm{B:C} 1.4$ top, (e) $550^{\circ} \mathrm{C} \mathrm{B:C} 1.4$ top, and (f) $600^{\circ} \mathrm{C}$ B:C 1.4 top.

Figure S8. Effect of $\mathrm{B}: \mathrm{C}$ on aromatic $\mathrm{C}-\mathrm{H}$ bonds in the HSQC NMR spectra for (a) $550^{\circ} \mathrm{C} \mathrm{B}: \mathrm{C} 1.0$ bottom, (b) $550^{\circ} \mathrm{C}$ B:C 1.4 bottom, (c) $550^{\circ} \mathrm{C} \mathrm{B:C} 1.8$ bottom, (d) $550^{\circ} \mathrm{C} \mathrm{B:C} 1.0$ top, (e) $550^{\circ} \mathrm{C} \mathrm{B:C} 1.4$ top, and (f) $550^{\circ} \mathrm{C}$ $\mathrm{B}: \mathrm{C} 1.8$ top.

Figure S9. Effect of catalytic upgrading temperature on methoxyl group in the HSQC NMR spectra for (a) $500^{\circ} \mathrm{C}$ B:C 1.3 bottom, (b) $550^{\circ} \mathrm{C} \mathrm{B:C} 1.3$ bottom, (c) $600^{\circ} \mathrm{C} \mathrm{B:C} 1.3$ bottom, (d) $500^{\circ} \mathrm{C} \mathrm{B:C} 1.3$ top, (e) $550^{\circ} \mathrm{C} \mathrm{B:C} 1.3$ top, and (f) $600^{\circ} \mathrm{C} \mathrm{B:C} 1.3$ top.

Figure S10. Effect of B:C ratio on methoxyl group in the HSQC NMR spectra for (a) $550^{\circ} \mathrm{C} \mathrm{B:C} 1.0$ bottom, (b) $550^{\circ} \mathrm{C}$ B:C 1.4 bottom, (c) $550^{\circ} \mathrm{C} \mathrm{B:C} 1.8$ bottom, (d) $550^{\circ} \mathrm{C} \mathrm{B:C} 1.0$ top, (e) $550^{\circ} \mathrm{C} \mathrm{B:C} 1.4$ top, and (f) $550^{\circ} \mathrm{C}$ $\mathrm{B}: \mathrm{C} 1.8$ top.

\section{Tables}

Table S1. Mass and carbon yields based on biomass feed.

Table S2. Effect of B:C ratio and upgrading temperature on MW distribution of bio-oil.

Table S3. Effect of CFP upgrading temperature and effect of B:C ratio on product yield distribution and bio-oil composition. 
XRD Profiles: Intensity vs. two-theta (degrees)

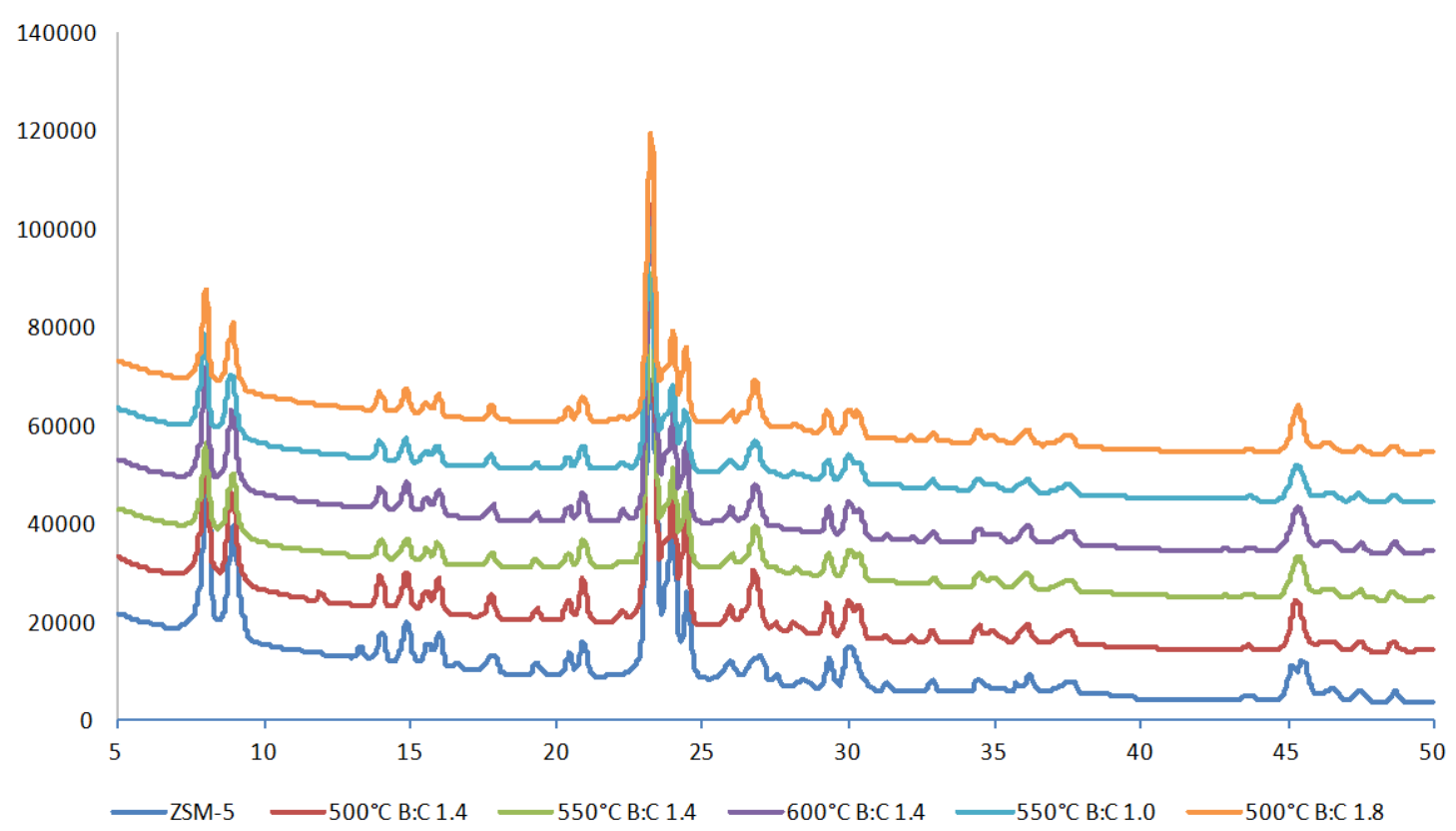

Figure S1. XRD analysis of the fresh ZSM-5 catalyst and post-reaction catalysts from the CFP experiments. 


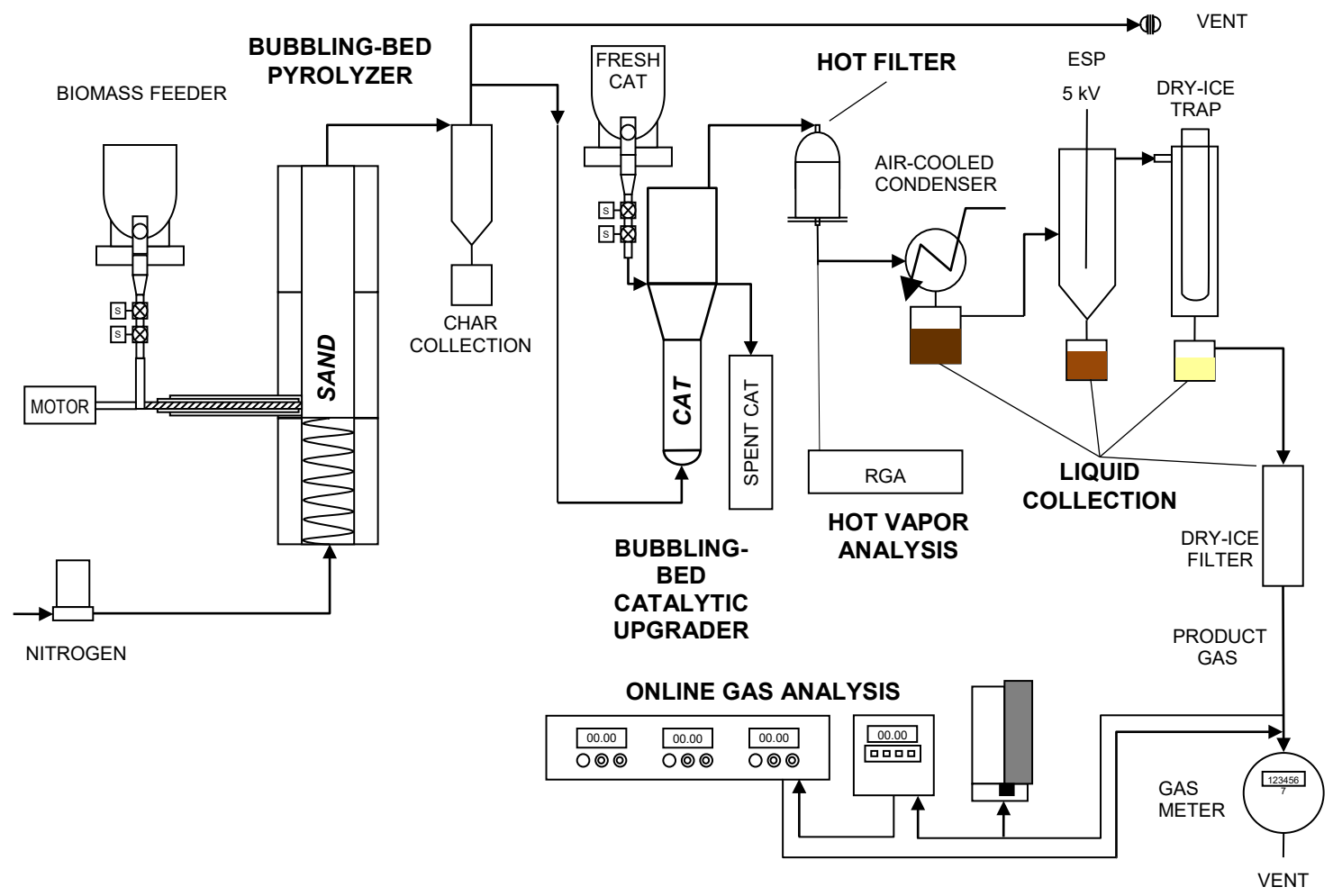

Figure S2. 2-inch fluidized bed reactor system with ex situ upgrading with continuous catalyst feed and removal. 


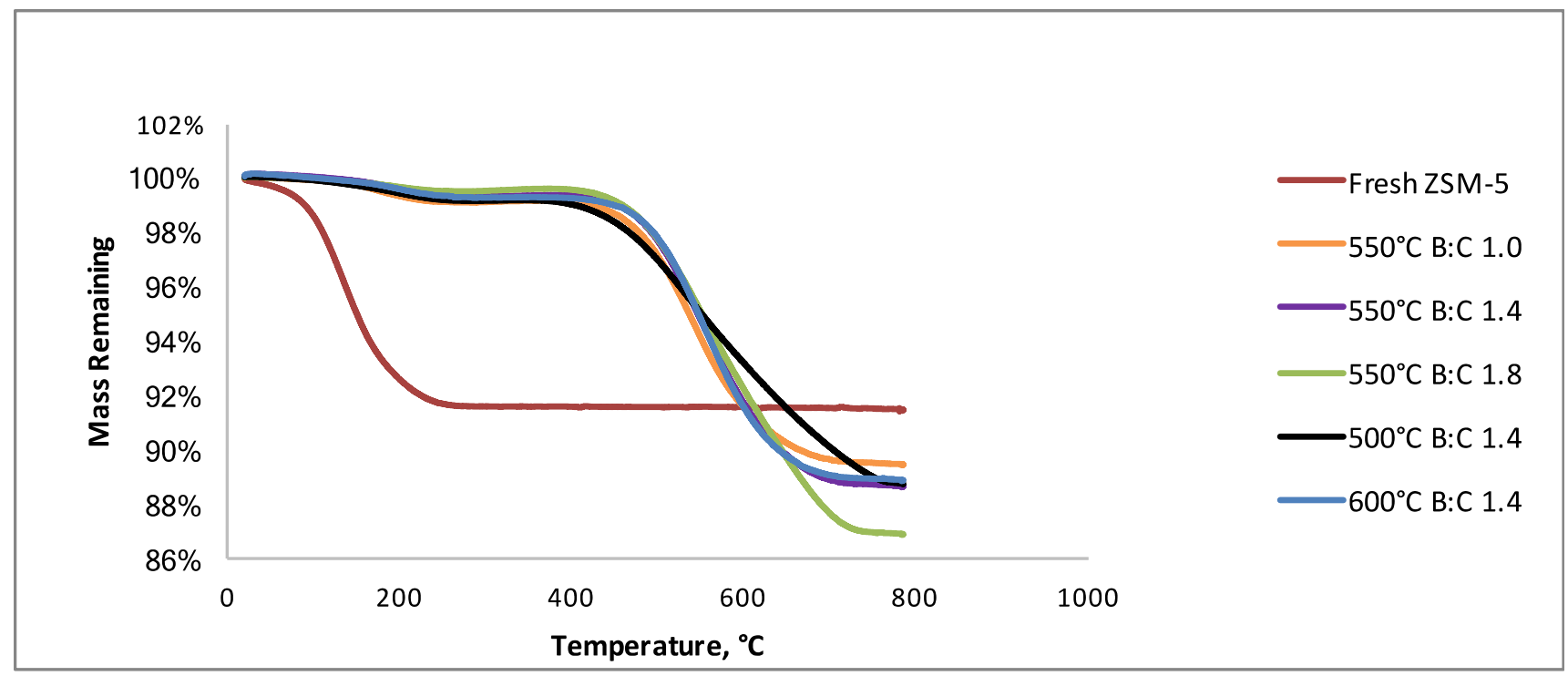

Figure S3. TGA traces for fresh catalyst and coked catalysts. The fresh catalyst shows the presence of $8 \%$ of water or other volatile material. For the coked catalysts, the mass loss up to $\sim 250^{\circ} \mathrm{C}$ was attributed to water and other loosely adsorbed species, and the mass loss after $650^{\circ} \mathrm{C}$ to coke. 

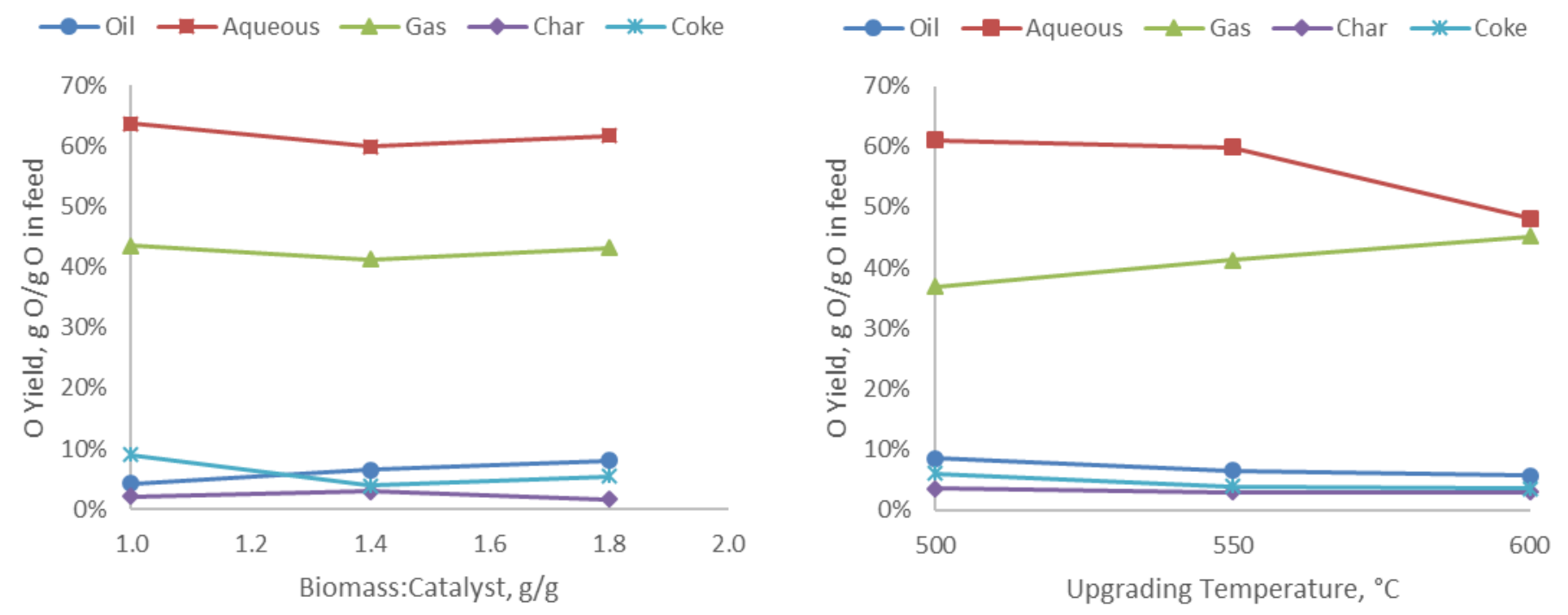

Figure S4. Yield of oxygen in the CFP products as a function of a) biomass:catalyst mass ratio and b) upgrading temperature. 

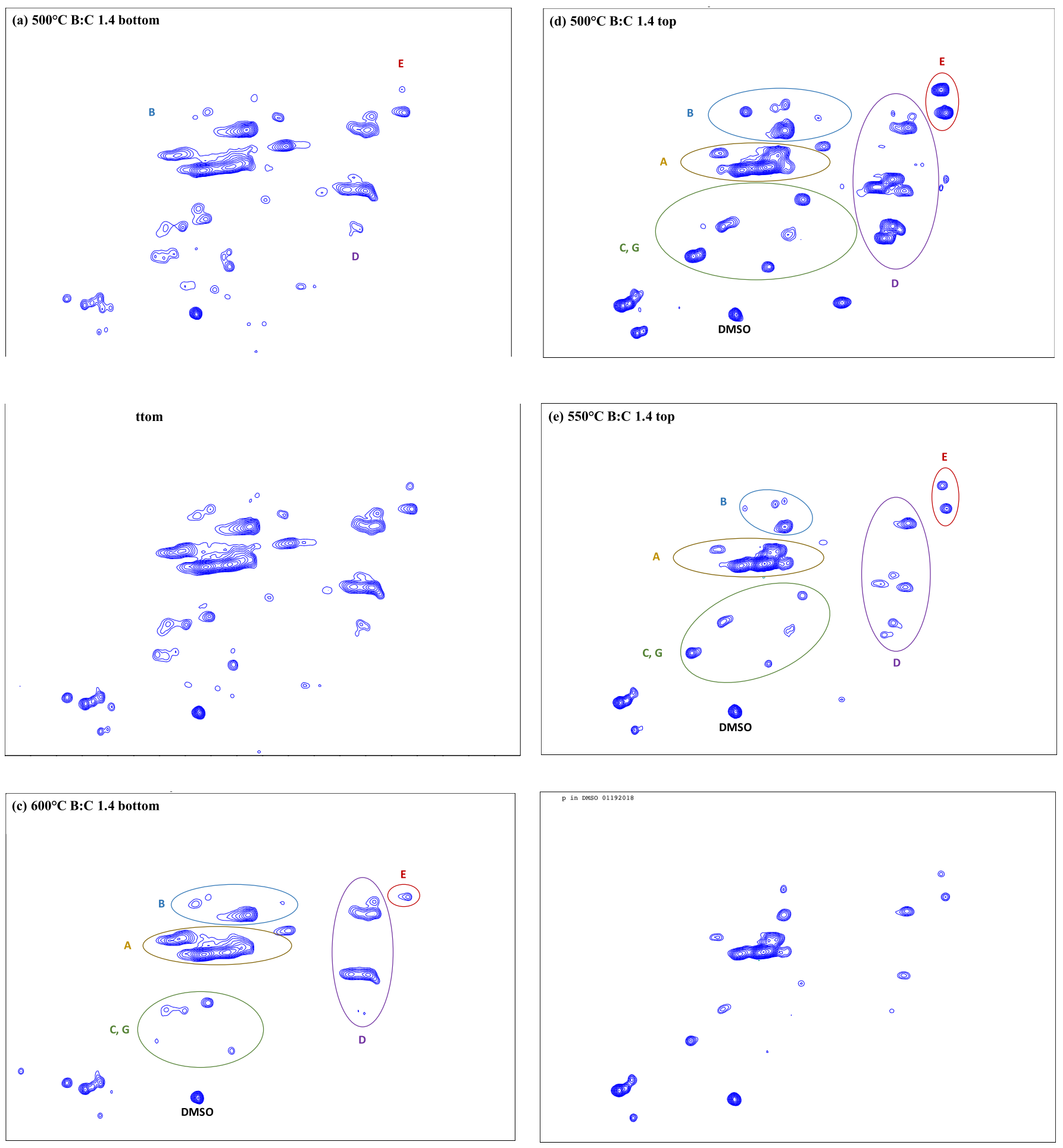

Figure S5. Effect of upgrading temperature on aliphatic $\mathrm{C}-\mathrm{H}$ bonds in the HSQC NMR spectra for (a) $500^{\circ} \mathrm{C} \mathrm{B}$ : C 1.4 bottom, (b) $550^{\circ} \mathrm{C} \mathrm{B:C} 1.4$ bottom, (c) $600^{\circ} \mathrm{C} \mathrm{B:C} 1.4$ bottom, (d) $500^{\circ} \mathrm{C} \mathrm{B:C} 1.4$ top, (e) $550^{\circ} \mathrm{C}$ B:C 1.4 top, and (f) $600^{\circ} \mathrm{C}$ B:C 1.4 top. 

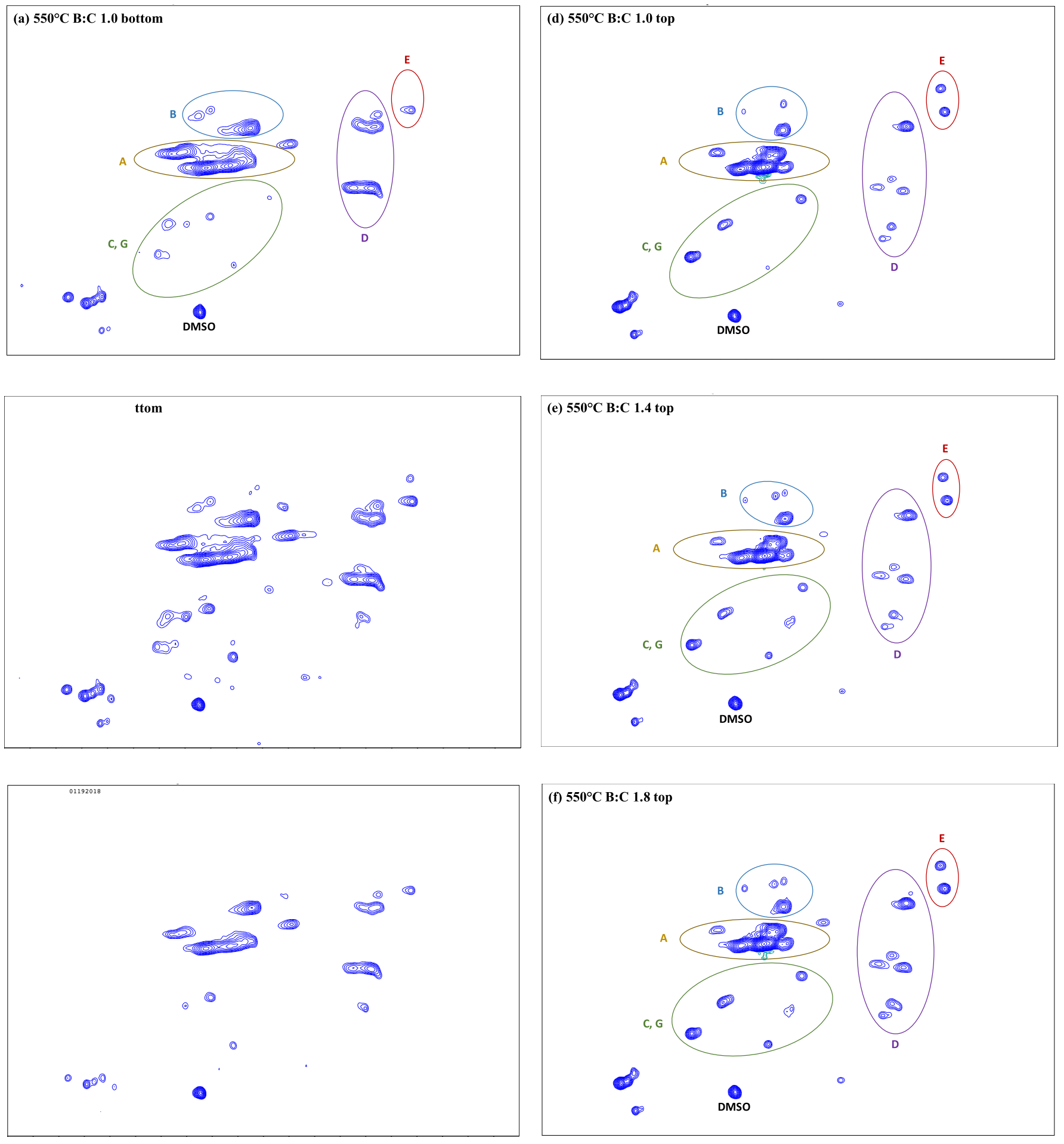

Figure S6. Effect of B:C ratio on aliphatic C-H bonds in the HSQC NMR spectra for (a) $550^{\circ} \mathrm{C} \mathrm{B:C} 1.0$ bottom, (b) $550^{\circ} \mathrm{C} \mathrm{B:C} 1.4$ bottom, (c) $550^{\circ} \mathrm{C} \mathrm{B:C} 1.8$ bottom, (d) $550^{\circ} \mathrm{C} \mathrm{B:C} 1.0$ top, (e) $550^{\circ} \mathrm{C}$ B:C 1.4 top, and (f) $550^{\circ} \mathrm{C}$ B:C 1.8 top. 

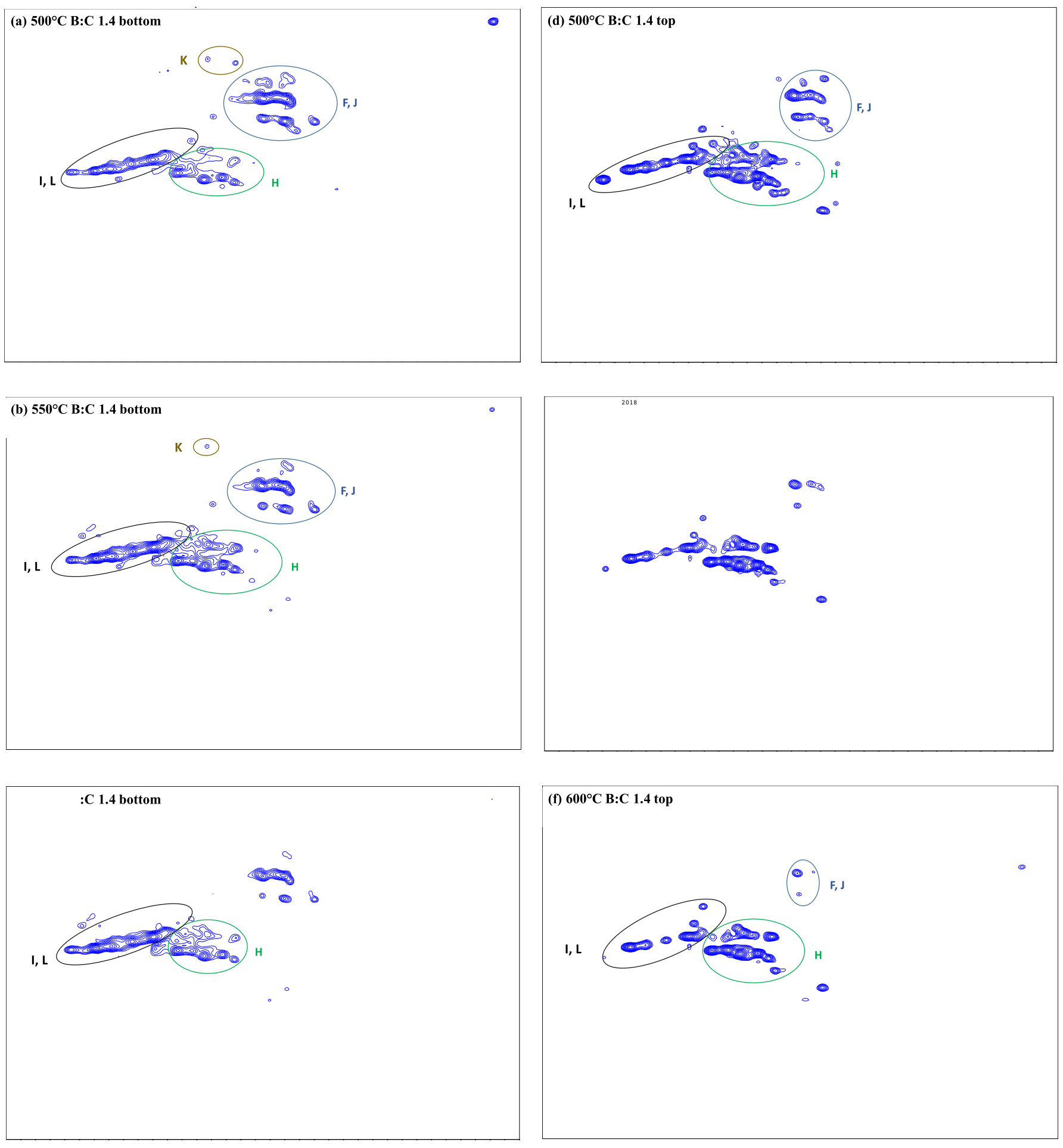

Figure S7. Effect of upgrading temperature on aromatic C-H bonds in the HSQC NMR spectra for (a) $500^{\circ} \mathrm{C} \mathrm{B:C}$ 1.4 bottom, (b) $550^{\circ} \mathrm{C} \mathrm{B:C} 1.4$ bottom, (c) $600^{\circ} \mathrm{C}$ B:C 1.4 bottom, (d) $500^{\circ} \mathrm{C} \mathrm{B:C} 1.4$ top, (e) $550^{\circ} \mathrm{C} \mathrm{B:C} 1.4$ top, and (f) $600^{\circ} \mathrm{C}$ B:C 1.4 top. 

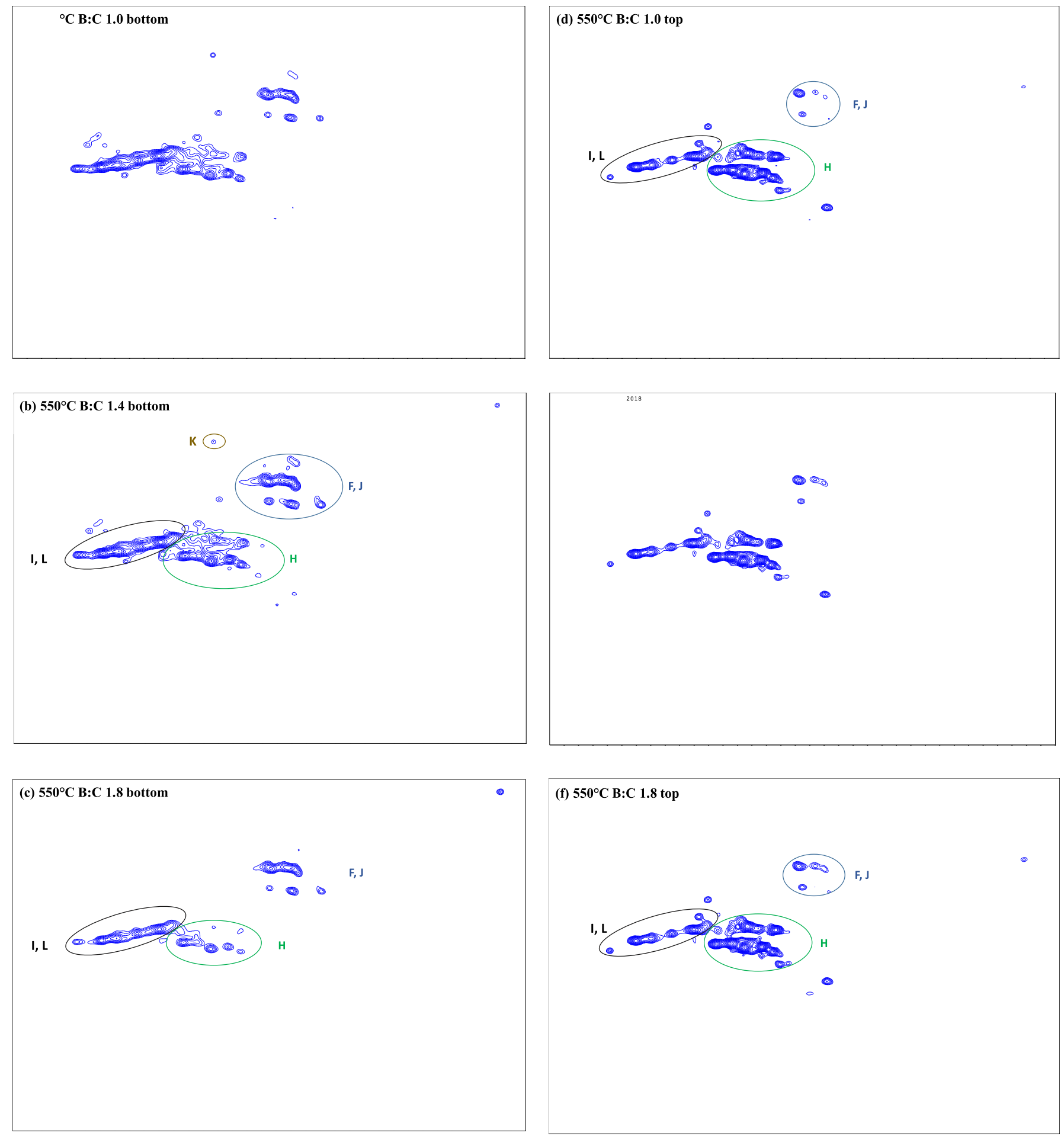

Figure S8. Effect of B:C on aromatic C-H bonds in the HSQC NMR spectra for (a) $550^{\circ} \mathrm{C} \mathrm{B:C} 1.0$ bottom, (b) $550^{\circ} \mathrm{C}$ B:C 1.4 bottom, (c) $550^{\circ} \mathrm{C} \mathrm{B:C} 1.8$ bottom, (d) $550^{\circ} \mathrm{C} \mathrm{B:C} 1.0$ top, (e) $550^{\circ} \mathrm{C} \mathrm{B:C} 1.4$ top, and (f) $550^{\circ} \mathrm{C}$ $\mathrm{B}: \mathrm{C} 1.8$ top. 

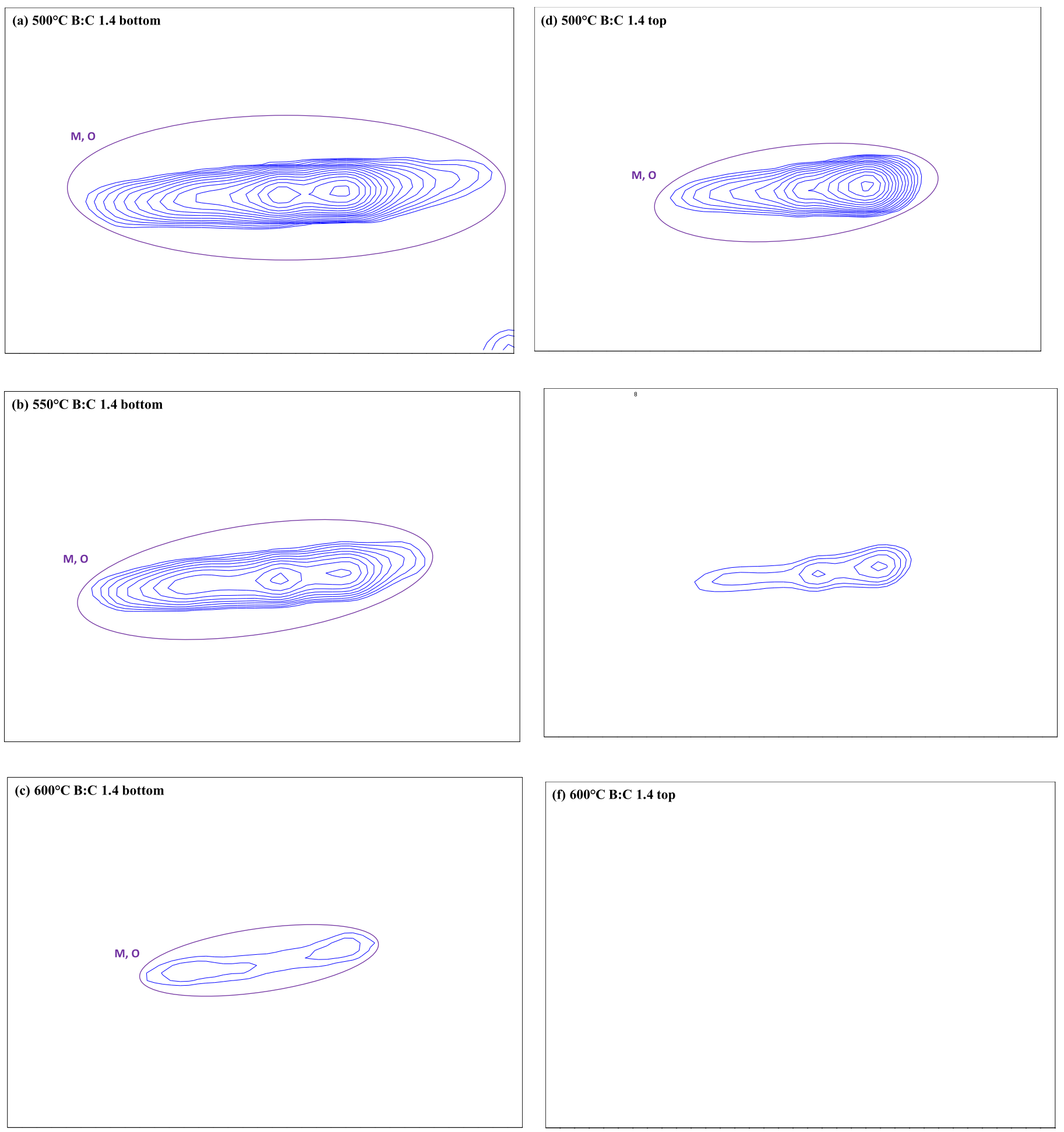

Figure S9. Effect of catalytic upgrading temperature on methoxyl group in the HSQC NMR spectra for (a) $500^{\circ} \mathrm{C}$ B:C 1.3 bottom, (b) $550^{\circ} \mathrm{C} \mathrm{B:C} 1.3$ bottom, (c) $600^{\circ} \mathrm{C} \mathrm{B:C} 1.3$ bottom, (d) $500^{\circ} \mathrm{C} \mathrm{B:C} 1.3$ top, (e) $550^{\circ} \mathrm{C} \mathrm{B:C} 1.3$ top, and (f) $600^{\circ} \mathrm{C} \mathrm{B:C} 1.3$ top. 

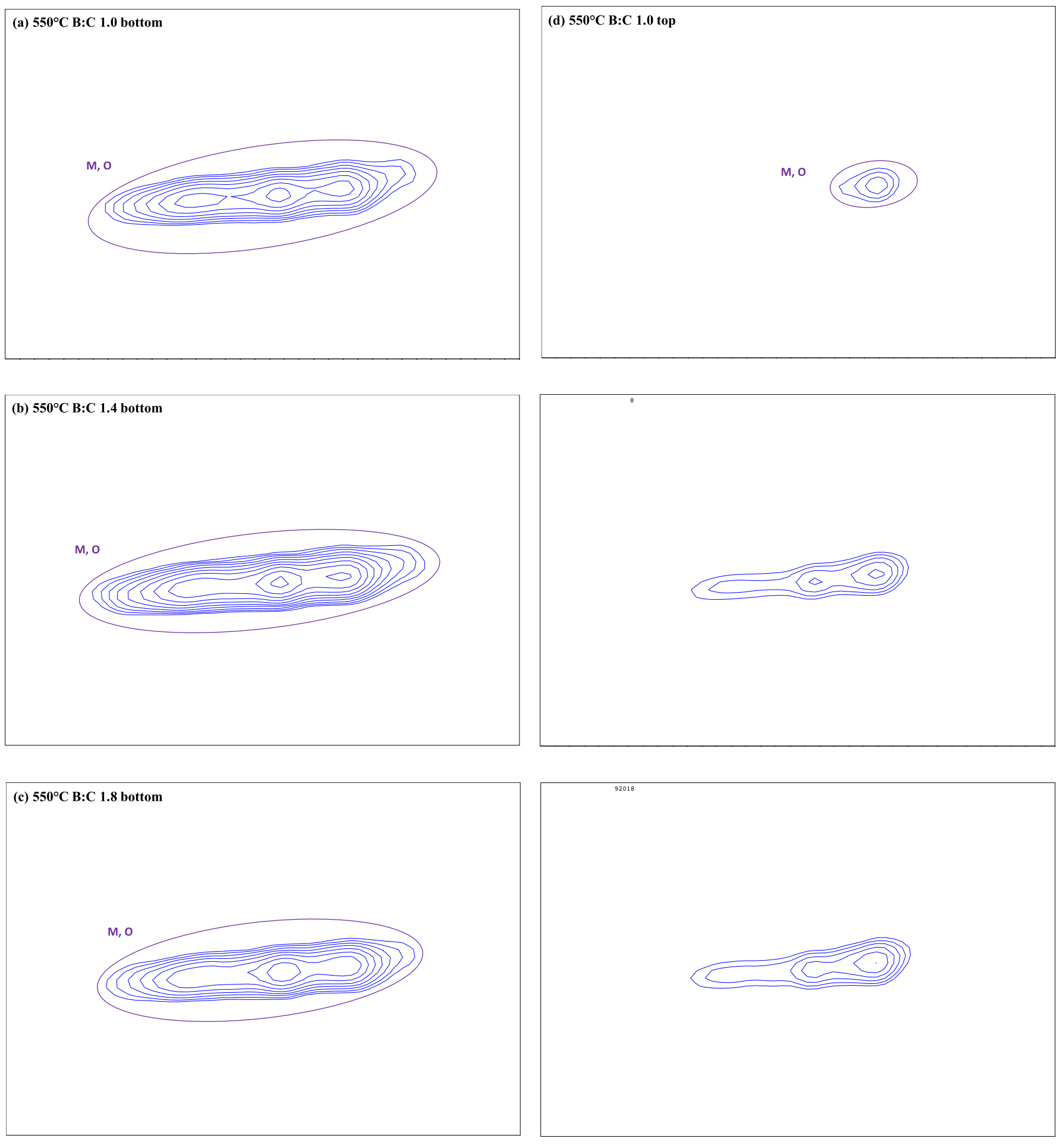

Figure S10. Effect of B:C ratio on methoxyl group in the HSQC NMR spectra for (a) 550 ${ }^{\circ} \mathrm{C}: \mathrm{C} 1.0$ bottom, (b) $550^{\circ} \mathrm{C} \mathrm{B:C} 1.4$ bottom, (c) $550^{\circ} \mathrm{C} \mathrm{B:C} 1.8$ bottom, (d) $550^{\circ} \mathrm{C} \mathrm{B:C} 1.0$ top, (e) $550^{\circ} \mathrm{C} \mathrm{B:C} 1.4$ top, and (f) $550^{\circ} \mathrm{C}$ $\mathrm{B}: \mathrm{C} 1.8$ top. 
Table S1. Mass and carbon yields based on biomass feed.

\begin{tabular}{|c|c|c|c|c|c|c|c|c|}
\hline & CFP & CFP & CFP & CFP & CFP* & CFP* & CFP & FP* \\
\hline \multirow{2}{*}{$\begin{array}{l}\text { Upgrading } \mathrm{T},{ }^{\circ} \mathrm{C} \\
\text { Biomass:Catalyst, } \\
\mathrm{g} / \mathrm{g}\end{array}$} & 550 & 550 & 550 & 550 & 500 & 600 & 600 & $\mathrm{n} / \mathrm{a}$ \\
\hline & 1.0 & 1.4 & 1.4 & 1.8 & 1.4 & 1.4 & 1.4 & $\mathrm{n} / \mathrm{a}$ \\
\hline \multicolumn{9}{|l|}{ Yields, g/g biomass } \\
\hline Oil & $13.4 \%$ & $15.2 \%$ & $16.4 \%$ & $17.7 \%$ & $17.1 \%$ & $14.4 \%$ & $13.2 \%$ & $73.9 \%$ \\
\hline Aqueous & $25.1 \%$ & $25.7 \%$ & $26.0 \%$ & $28.4 \%$ & $26.3 \% *$ & $19.9 \% *$ & $25.3 \%$ & $\mathrm{n} / \mathrm{a}^{* *}$ \\
\hline Gas & $35.9 \%$ & $34.4 \%$ & $33.2 \%$ & $35.5 \%$ & $29.5 \%$ & $37.7 \%$ & $39.2 \%$ & $12.3 \%$ \\
\hline Char & $9.8 \%$ & $9.8 \%$ & $10.1 \%$ & $9.8 \%$ & $9.3 \%$ & $9.1 \%$ & $9.8 \%$ & $9.2 \%$ \\
\hline Coke & $10.2 \%$ & $8.9 \%$ & $8.2 \%$ & $7.5 \%$ & $8.5 \%$ & $7.4 \%$ & $8.1 \%$ & $0.0 \%$ \\
\hline Total & $94.2 \%$ & $93.9 \%$ & $93.8 \%$ & $98.7 \%$ & $90.5 \%$ & $88.2 \%$ & $95.4 \%$ & $95.3 \%$ \\
\hline \multicolumn{9}{|c|}{ Carbon Yields, g C/g C in biomass } \\
\hline Oil & $21.2 \%$ & $23.7 \%$ & $24.9 \%$ & $26.0 \%$ & $25.1 \%$ & $21.8 \%$ & $20.7 \%$ & $70.9 \%$ \\
\hline Aqueous & $0.9 \%$ & $2.3 \%$ & $2.4 \%$ & $2.9 \%$ & $3.4 \%$ & $1.7 \%$ & $4.0 \%$ & $\mathrm{n} / \mathrm{a} * *$ \\
\hline Gas & $32.4 \%$ & $31.0 \%$ & $29.7 \%$ & $31.9 \%$ & $26.0 \%$ & $34.3 \%$ & $35.6 \%$ & $9.5 \%$ \\
\hline Char & $16.5 \%$ & $16.1 \%$ & $16.7 \%$ & $16.7 \%$ & $15.0 \%$ & $15.0 \%$ & $16.2 \%$ & $15.4 \%$ \\
\hline Coke & $15.9 \%$ & $13.1 \%$ & $12.0 \%$ & $12.7 \%$ & $13.8 \%$ & $12.7 \%$ & $12.3 \%$ & $0.0 \%$ \\
\hline Total & $86.9 \%$ & $86.1 \%$ & $85.7 \%$ & $90.3 \%$ & $83.2 \%$ & $85.5 \%$ & $88.9 \%$ & $95.8 \%$ \\
\hline \multicolumn{9}{|l|}{ Gas Yields, $\mathrm{g} / \mathrm{g}$ feed } \\
\hline $\mathrm{H}_{2}$ & $0.2 \%$ & $0.2 \%$ & $0.2 \%$ & $0.2 \%$ & $0.1 \%$ & $0.3 \%$ & $0.3 \%$ & $0.1 \%$ \\
\hline $\mathrm{CH}_{4}$ & $1.3 \%$ & $1.3 \%$ & $1.3 \%$ & $1.4 \%$ & $0.9 \%$ & $1.7 \%$ & $1.8 \%$ & $0.5 \%$ \\
\hline $\mathrm{CO}$ & $18.1 \%$ & $17.8 \%$ & $16.8 \%$ & $18.1 \%$ & $15.5 \%$ & $19.7 \%$ & $20.2 \%$ & $5.5 \%$ \\
\hline $\mathrm{CO}_{2}$ & $11.2 \%$ & $10.6 \%$ & $10.6 \%$ & $11.0 \%$ & $9.5 \%$ & $11.0 \%$ & $11.6 \%$ & $5.7 \%$ \\
\hline $\mathrm{C}_{2}-\mathrm{C}_{4}$ olefins & $5.0 \%$ & $4.5 \%$ & $4.4 \%$ & $4.7 \%$ & $3.5 \%$ & $5.0 \%$ & $5.3 \%$ & $0.4 \%$ \\
\hline $\mathrm{CO}: \mathrm{CO}_{2}, \mathrm{~mol} / \mathrm{mol}$ & 2.53 & 2.65 & 2.50 & 2.58 & 2.57 & 2.80 & 2.73 & 1.53 \\
\hline \multicolumn{9}{|c|}{ Gas Carbon Yields, g/g feed } \\
\hline $\mathrm{CH}_{4}$ & $2.0 \%$ & $2.0 \%$ & $1.9 \%$ & $2.2 \%$ & $1.4 \%$ & $2.5 \%$ & $2.7 \%$ & $0.8 \%$ \\
\hline $\mathrm{CO}$ & $15.7 \%$ & $15.4 \%$ & $14.5 \%$ & $15.7 \%$ & $13.4 \%$ & $17.0 \%$ & $17.4 \%$ & $4.8 \%$ \\
\hline $\mathrm{CO}_{2}$ & $6.2 \%$ & $5.8 \%$ & $5.8 \%$ & $6.1 \%$ & $5.2 \%$ & $6.1 \%$ & $6.4 \%$ & $3.1 \%$ \\
\hline $\mathrm{C}_{2}-\mathrm{C}_{4}$ olefins & $8.7 \%$ & $7.9 \%$ & $7.6 \%$ & $8.1 \%$ & $6.1 \%$ & $8.7 \%$ & $9.2 \%$ & $0.7 \%$ \\
\hline
\end{tabular}

* These experiments were performed with 1 dry-ice trap vs. 2 in the other experiments. The dry ice-traps collect mainly aqueous liquid; a comparison of the experiments at $600^{\circ} \mathrm{C}$ with 1 and 2 dry-ice traps shows that the addition of the second trap increased the aqueous phase yield but not the oil yield.

** Not applicable. There is no separate aqueous phase in the non-catalytic fast pyrolysis experiment. 
Table S2. Effect of B:C ratio and upgrading temperature on MW distribution of bio-oil.

\begin{tabular}{|c|c|c|c|c|}
\hline \multirow{2}{*}{\multicolumn{2}{|c|}{ Fffect of $\mathrm{B} \cdot \mathrm{C}$ ratio }} & $\mathrm{M}_{\mathrm{n}}$ & $\mathrm{M}_{\mathrm{w}}$ & PDI \\
\hline & \multicolumn{4}{|c|}{ Effect of $\mathrm{B}: \mathrm{C}$ ratio } \\
\hline & FP & 289 & 488 & 1.69 \\
\hline \multirow{3}{*}{ Bottom } & $550^{\circ} \mathrm{C} \mathrm{B}: \mathrm{C} 1.8$ & 132 & 297 & 2.25 \\
\hline & $550^{\circ} \mathrm{C} \mathrm{B}: \mathrm{C} 1.4$ & 120 & 251 & 2.08 \\
\hline & $550^{\circ} \mathrm{C} \mathrm{B}: \mathrm{C} 1.0$ & 108 & 197 & 1.82 \\
\hline \multirow{3}{*}{ Top } & $550^{\circ} \mathrm{C} \mathrm{B}: \mathrm{C} 1.8$ & 94.5 & 141 & 1.48 \\
\hline & $550^{\circ} \mathrm{C} \mathrm{B}: \mathrm{C} 1.4$ & 98.8 & 161 & 1.63 \\
\hline & $550^{\circ} \mathrm{C} \mathrm{B}: \mathrm{C} 1.0$ & 90.0 & 124 & 1.38 \\
\hline \multicolumn{5}{|c|}{ Effect of upgrading temperature } \\
\hline \multirow{3}{*}{ Bottom } & $500^{\circ} \mathrm{C} \mathrm{B}: \mathrm{C} 1.4$ & 131 & 291 & 2.22 \\
\hline & $550^{\circ} \mathrm{C} \mathrm{B}: \mathrm{C} 1.4$ & 120 & 251 & 2.08 \\
\hline & $600^{\circ} \mathrm{C} \mathrm{B}: \mathrm{C} 1.4$ & 112 & 219 & 1.96 \\
\hline \multirow{3}{*}{ Top } & $500^{\circ} \mathrm{C} \mathrm{B}: \mathrm{C} 1.4$ & 96.1 & 165 & 1.71 \\
\hline & $550^{\circ} \mathrm{C} \mathrm{B}: \mathrm{C} 1.4$ & 98.8 & 161 & 1.63 \\
\hline & $600^{\circ} \mathrm{C} \mathrm{B}: \mathrm{C} 1.4$ & 86.6 & 122 & 1.41 \\
\hline
\end{tabular}


Table S3. Effect of CFP upgrading temperature and effect of B:C ratio on product yield distribution and bio-oil composition.

Effect of CFP upgrading temperature

Bio-oil yield, O content in CFP bio-oil, $\mathrm{C}$ yield in Coke $\mathrm{C}$ yield, indenols/naphthols, 1-ring HC, 2aqueous phase, coke $\mathrm{C}$ yield, methoxyphenols, ring $\mathrm{HC}, 3+4$-ring $\mathrm{HC}$, molecular homogeneity indenols/naphthols, phenols

Decreases with increasing temperature

Gas $\mathrm{C}$ yield, $\mathrm{CO}, \mathrm{CH}_{4}$, light alkenes, 1-ring $\mathrm{HC}$, 2ring $\mathrm{HC}$, 3+4-ring $\mathrm{HC}$, molecular homogeneity

Increases with increasing temperature

$\mathrm{CO}_{2}$ yield

No clear trend Decreases with increasing B:C ratio

\section{Effect of B:C ratio}

ring $\mathrm{HC}$, 3+4-ring $\mathrm{HC}$, molecular homogeneity

Bio-oil yield, O content in CFP bio-oil, C yield in aqueous phase, phenols, levoglucosan

Increases with increasing $\mathrm{B}: \mathrm{C}$ ratio

Gas C yield, CO, light alkenes, methoxyphenols

No clear trend 